\title{
Composite CDE: modeling composite relationships between common data elements for representing complex clinical data
}

Hye Hyeon Kim', Yu Rang Park ${ }^{2}$, Suehyun Lee ${ }^{3}$ and Ju Han Kim ${ }^{1 *}$ (D)

\begin{abstract}
Background: Semantic interoperability is essential for improving data quality and sharing. The ISO/IEC 11179 Metadata Registry (MDR) standard has been highlighted as a solution for standardizing and registering clinical data elements (DEs). However, the standard model has both structural and semantic limitations, and the number of DEs continues to increase due to poor term reusability. Semantic types and constraints are lacking for comprehensively describing and evaluating DEs on real-world clinical documents.
\end{abstract}

Methods: We addressed these limitations by defining three new types of semantic relationship (dependency, composite, and variable) in our previous studies. The present study created new and further extended existing semantic types (hybrid atomic and repeated and dictionary composite common data elements [CDEs]) with four constraints: ordered, operated, required, and dependent. For evaluation, we extracted all atomic and composite CDEs from five major clinical documents from five teaching hospitals in Korea, 14 Fast Healthcare Interoperability Resources (FHIR) resources from FHIR bulk sample data, and MIMIC-III (Medical Information Mart for Intensive Care) demo dataset. Metadata reusability and semantic interoperability in real clinical settings were comprehensively evaluated by applying the CDEs with our extended semantic types and constraints.

Results: All of the CDEs $(n=1142)$ extracted from the 25 clinical documents were successfully integrated with a very high CDE reuse ratio (46.9\%) into 586 CDEs (259 atomic and 20 unique composite CDEs), and all of CDEs ( $n=$ 238) extracted from the 14 FHIR resources of FHIR bulk sample data were successfully integrated with high CDE reuse ration (59.7\%) into 96 CDEs (21 atomic and 28 unique composite CDEs), which improved the semantic integrity and interoperability without any semantic loss. Moreover, the most complex data structures from two CDE projects were successfully encoded with rich semantics and semantic integrity.

Conclusion: MDR-based extended semantic types and constraints can facilitate comprehensive representation of clinical documents with rich semantics, and improved semantic interoperability without semantic loss.

Keywords: Common data elements, Semantic interoperability, Semantic relationship, Metadata registry

\footnotetext{
* Correspondence: juhan@snu.ac.kr

${ }^{1}$ Seoul National University Biomedical Informatics (SNUBI), Division of

Biomedical Informatics, Seoul National University College of Medicine, Seoul

03080, Republic of Korea

Full list of author information is available at the end of the article
}

C The Author(s). 2020 Open Access This article is licensed under a Creative Commons Attribution 4.0 International License, which permits use, sharing, adaptation, distribution and reproduction in any medium or format, as long as you give appropriate credit to the original author(s) and the source, provide a link to the Creative Commons licence, and indicate if changes were made. The images or other third party material in this article are included in the article's Creative Commons licence, unless indicated otherwise in a credit line to the material. If material is not included in the article's Creative Commons licence and your intended use is not permitted by statutory regulation or exceeds the permitted use, you will need to obtain permission directly from the copyright holder. To view a copy of this licence, visit http://creativecommons.org/licenses/by/4.0/. The Creative Commons Public Domain Dedication waiver (http://creativecommons.org/publicdomain/zero/1.0/) applies to the data made available in this article, unless otherwise stated in a credit line to the data. 


\section{Background}

Data harmonization and interoperability are essential for advancing biomedical research. These features can be achieved by representing clinical data in a standard format, and they are crucial for facilitating understanding and sharing data across diverse translational studies [1,2]. A data element (DE) is defined as the fundamental unit of data which contains information with a clear conceptualized meaning, together with its representation, and is considered as the correct approach for standardizing data and improving data quality (DQ) and efficiency.

The ISO/IEC 11179 Metadata Registry (MDR) standard describes a method of standardizing and registering DEs to make them understandable and shareable between studies and institutions. MDR-based DE provide data uniformly and interoperability between clinical studies and institutions since they are specified based on a standard metadata model that consists of a sets of attributes, which are delineating the definition, identification, representation, classification, and permissible values [3-5].

The terms DE and common data element (CDE) have been used interchangeably in many ways. However, it can be clearly explained by defining these two terms as the following. The term DE is an atomic unit of data that has precise meaning and precise semantics in metadata. CDE is a data element that is common to multiple data sets across different studies [6]. In this paper, we used the term DE to specifically describe the concept of metadata, but for all other cases we used the term CDE.

CDEs are increasingly being used by clinical researchers in trials for harmonizing data collected across diverse studies. The use of standardized CDEs provides various benefits to investigators including (1) rapid and efficient study start-up by enabling access to defined CDEs and case report forms (CRFs) and (2) enriched data sharing and aggregation using standard definitions and forms [7].

The use of CDEs has been extended to clinical practice by using standardized CDEs for representing the clinical information in electronic health records (EHRs). For example, Newton et al. included phenotype data in EHRs using CDEs to facilitate EHR-driven genomic studies [8]. The National Institutes of Health have developed ISO/ IEC 11179 MDR-based CDEs providing a controlled terminology for data descriptors. They also encouraged clinical researchers to use CDEs to facilitate data harmonization [5]. CDEs have been adopted in numerous clinical domains including cancer, stroke, epilepsy, rare disease, emergency medicine, and radiology for patient care and research. Utilizing CDEs will facilitate secondary data use (i.e., 'collect once and use many times'), which is an approach to data standardization for spanning silos in primary and secondary data use [9].

However, ISO/IEC 11179 MDR focuses only on the representation of individual and independent CDEs without providing the ability to describe constraints for a CDE nor relationships among different CDEs, which are essential for fully describe, semantically compose, and correctly interpret CDEs of clinical documents [10-13]. Although ISO/IEC 11179 MDR standard describes Derived Data Element (DDE) [14] detailing the relationship between a $\mathrm{CDE}$ and another $\mathrm{CDE}$ from which it is derived with the rule controlling its derivation, this approach is inherently limited by requiring one or more input $\mathrm{CDEs}$ and the DDE becoming output DE. For example, while CDEs for describing systolic blood pressure (SBP) and diastolic blood pressure (DBP) can be easily defined as two separate ones annotated with standardized metadata conforming to the ISO/IEC 11179 MDR standard, these two CDEs become mere input CDEs and a separate output CDE should be created as the DDE. Also, a constraint between the two CDEs such as 'the SBP must be greater than the DBP' is usually described outside of the CDEs for there is no designated reason for the CDEs to carry constraint information.

To address these challenges in our previous study [10], we proposed three types of semantic relationships (i.e, variable, dependency, and composite relationships) representing semantic constraints or rules among multiple CDEs. These relationships can be described as follows: First, CDEs are in a variable relationship when they can be systematically derived from a base CDE by applying a standardized concept from a controlled vocabulary as the variable. For example, the meanings of two CDEs for 'normal value range of laboratory test, Albumin' and 'normal value range of laboratory test, Homocysteine' are closely related, differing only in the laboratory test names of 'Albumin' and 'Homocysteine.' It means many lab tests related CDEs can be assigned to one variable $\mathrm{CDE}$. The variable relationship can systematically represent all these variations as a single CDE, 'DE: Normal value range of lab test $x$,' by specifying a controlled vocabulary such as LOINC. The variable relationship can therefore systematically reduce the number of required CDEs. Second, a CDE is in a dependency relationship may influence the possible determinations of the value space of the $\operatorname{CDE}(\mathrm{s})$ base on the value of another $\mathrm{CDE}(\mathrm{s})$. For example, the value of a certain $\mathrm{CDE}$ may be defined as the sum of the values of a set of CDEs in a questionnaire. Third, the composite relationship can be conveniently applied to integrate several interrelated CDEs into a composite CDE. For example, the medical history of a patient is likely to be more informative when body parts are correctly assigned, which can be achieved by grouping 'DE: Body System for Medical History' and 'DE: Medical History Specify' into the composite CDE of 'DE: Medical History.' However, we realized that our previous work, supports relatively simple semantic relationships among CDEs and is not robust enough to 
cover many other specific challenges associated with CDEs used in real-world clinical forms.

The present study further proposes extended semantic types (hybrid atomic CED (aCDE) and repeated and dictionary composite CDEs (cCDEs)) and four semantic constraints (ordered, operated, required, and dependent) for correctly representing even more complex but essential semantic relationships between CDEs that are found in real-world clinical documents (Fig. 1). We found useful patterns characterizing challenging cases, that required further semantic definitions and descriptions as the following four cases;

\section{Data entries with multiple data types}

A data type determines the type of data that can be entered and stored in a CDE and each CDE contains only one data type [15]. However, we found that free-textbased data entry in many clinical documents stored in EHRs often allows multiple data types to be entered and stored in the same attribute. For example, a laboratory result for syphilis normally has a numeric data type that allows numeric values (e.g., ' 0.8 ') as input. However, it often also requires the entry of string or logical data such as 'negative' or 'false' as input. Sometimes creating two strictly separate CDEs for the same laboratory result for syphilis (i.e., numeric and string) may cause more confusion than not. We found that sometimes it is better to allow either numeric or string data types for the same value domain. We created a value property (hybrid) to make it possible to ensure that conventional multiple data types are available in the same CDE by explicitly defining hybrid data type for a CDE.

\section{Dictionary data entries}

Data may refer to a controlled biomedical vocabulary for several reasons such as adherence to standards, semantic enrichment for better understanding, and input validation for improving semantic integrity. A CDE referring to a controlled biomedical vocabulary was defined as being in a variable relationship in our previous study [10]. We extended the concept of the variable relationship to dictionary data entries in order to tightly link a set of CDEs via a 'foreign key' between a real-world dictionary database and a controlled biomedical vocabulary. This also ensures that a set of CDEs and tuples with rich attributes provided by the dictionary are linked with their proper data type definitions and value domains.

\section{Tabular data entries with repeated data entry}

Clinical data are frequently described in tabular formats. A tabular data entry is an enclosed structure in which a composed set of CDEs is repetitively listed for repeated observations. For example, body weight and height may be measured for each patient when she/he visits for treatment. The set of data items such as body weight, height, and date of measurement should both be collected together and repeatedly. We created a value property (repeat) to ensure that the values that belong to the same set of CDEs are identified as such.

\section{Data constraints}

Highly interrelated CDEs in a clinical document need to be defined by semantic constraints for better interchange of semantics and context. By specifying constraints on an $\mathrm{aCDE}$, users can further narrow down the definition of what a valid value really means. For example, a derived value such as BMI (body mass index) can be automatically calculated from the values of the two aCDEs for body weight and height. Because the values of bodyweight and height aCDEs should not be null, a required constraint should be applied to each of the two aCDEs

\section{Composite relationship}
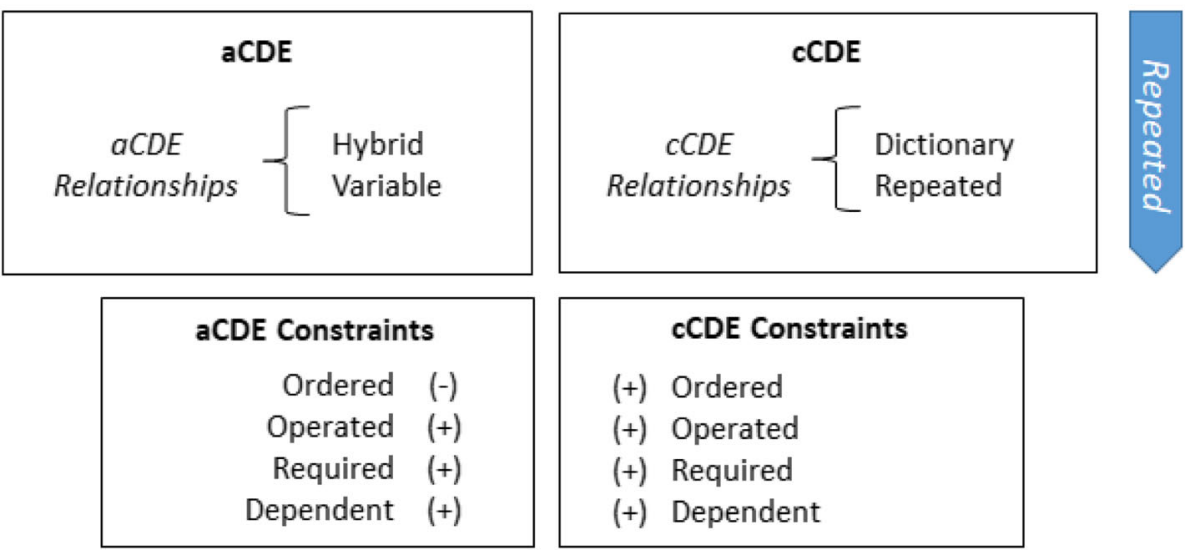

Fig. 1 Overview of the formal relationship between aCDE and CCDEs with extended semantic types and CDE-type specific constraints 
to make the BMI aCDE to be valid. The calculation formula to obtain BMI is described by an operated constraint of BMI aCDE.

For another example, for an aCDE related to the question of whether any drug side effect has happened with permissible answers of 'Yes' and 'No', the following aCDE, "Specify the drug side effect", holds only when the value was 'Yes'. These two aCDEs are in a dependent relationship with each other and the sequence of the two has an order. The dependent and sequence relationships can be defined by dependent and ordered constraints.

\section{Methods}

\section{Data sources: 2 CDE projects}

The National Institute of Neurological Disorders and Stroke (NINDS) CDE Project [16] is an ongoing effort to develop data standards for use in clinical research in neuroscience. It was initiated in 2006 to standardize data collection across neurological-disorder-related clinical studies funded by the NINDS. As of October 2016, the NINDS CDE project included 20 studies with 11,296 distinct CDEs. The NINDS CDEs are not fully compliant with ISO/IEC 11179. Instead they are provided with only simple CDE descriptions and definitions. However, a part of NIND CDEs that are registered in National Cancer Institute (NCI) cancer Data Standards Registry (caDSR) and reviewed by the NCI cancer Biomedical Informatics Grid project manager, conforms fully with the ISO/IEC 11179 MDR standard. In the present study, we used part of the NINDS CDEs, which are $308(3.1 \%)$ stroke and general CDEs of the NINDS in 57 CRFs (Supplementary Tables S1) that are registered in the caDSR. Selected CDEs within the context of their CRFs were explored for challenging cases requiring new semantic relationships that we have defined.

The DialysisNet and Avatar Beans Project is a tabletand phone-based mobile application developed by the Health Avatar Initiative [17]. The project started in 2013, and it has established clinical data standards for managing and harmonizing hemodialysis data across multiple medical institutions in Korea $[18,19]$. This project aims to improve the management of chronic kidney disease and end-stage renal disease by using an integrated mobile application for data collection and documentation. The DialysisNet application was initially built upon 122 distinct hemodialysis-associated CDEs based on CRFs from major four hemodialysis centers (Supplementary Tables S2). We used 11,428 CDEs from the above two projects for comprehensively defining and evaluating new $\mathrm{CDE}$ relationships and constraints.

\section{Designating key concepts}

The CRFs and clinical documents from the two CDE projects incorporate all the data collection items with CDEs. We first examined the CDEs to formalize the above mentioned four challenging cases. Figure 1 depics the formal relationships between atomic $(\mathrm{aCDE})$ and composite (cCDE) CDEs with type-specific constraints. Since the core structure of a CDE is a name-value pair augmented by $\mathrm{DE}$ concept-domain and value-domain details, an aCDE is a single unambiguously described data item [19]. Our previous and simple-minded definition of $\mathrm{CCDE}$ as a set of interrelated aCDEs [9] was extended to include two new semantic relationships: dictionary and repeated cCDEs.

For example, a drug side effect is regarded as an undesirable secondary effect that occurs in addition to the desired therapeutic effect of a medication. To correctly represent 'a drugs side effect', at least three types of information needs to be presented: 'drug name', 'drug dosage', 'drug side effect'. One can define the three types of information as aCDEs and then combine them to compose a cCDE.

We extracted aCDEs and cCDEs from the above mentioned two DE projects (NINDS and DialysisNet CDE Projects) and applied the extended semantic types and constraints. We then mapped and integrated the CDEs in order to comprehensively evaluate the metadata reusability and semantic interoperability in the clinicalpractice setting.

\section{Evaluation scheme}

For the purpose of evaluating the utility of the newly proposed semantic types and constraints, we used three different data sources: (1) deriving CDEs from clinical documents, (2) Fast Healthcare Interoperability Resources (FHIR) based structured data, and (3) practical clinical dataset from MIMIC-III (Medical Information Mart for Intensive Care).

For deriving CDEs from clinical docments, we collected 25 clinical documents in real-world clinical practice, comprising five documents including Admission Note, Initial Medical Examination Note, Discharge Summary, Emergency Note, and Operation Note from five major teaching hospitals in Korea: Seoul National University Hospital, Ajou University Medical Center, Pusan National University Hospital, Gachon University Gil Hospital, and Chonnam National University Hospital. It contains Patient, PastHistory, AdmissionInformation, Operation, FamilyHistory, SocialHistory, LabResult, Medication, VitalSign, Treatments, and PhysicalExam [18]. We chose these 25 clinical documents since these documents are used in common by all five hospitals and are essential in the process of patient admission to discharge, for representing the specificity of the data. The limits of these 25 clinical documents are their insufficiency in providing a richness of depth and detail concerning the levels of clinical data. Thus, we added two different structured data from the FHIR bulk sample data and the MIMIC-III demo dataset. 
FHIR is propagated as an open standard describing data formats and elements, known as 'resources' and an application programming interface (API) for exchanging EHR. FHIR's clinical resource definitions are concrete, intuitive concepts such as MedicationPrescription, AdverseReaction, Procedure, and Condition. The standard was created by the Health Level Seven International (HL7) healthcare standards organization. We downloaded FHIR bulk sample data, which is exported from a FHIR server to a preauthorized client by using FHIR bulk Downloader sample app [20-22]. Among 145 resources of FHIR version 4 [23], the FHIR bulk sample data contains 14 resources; AllergyIntolerance, CarePlan, Claim, Condition, Goal, Encounter, Observation, DiagnosticReport, Immunization, MedicationRequest, ImagingStudy, Organization, Patient, and Procedure. Although we could analyze metadata of all FHIR resources through the structural information provided by HL7, it was necessary to review the actual sample data with metadata to confirm the relationships and constraints among the data. Thus, we chose 14 out of the 145 FHIR resources.

The MIMIC-III clinical database contains comprehensive clinical data relating to tens of thousands of Intensive Care Unit patients. MIMIC-III is a large, freelyavailable database comprising of deidentified healthrelated data associated with over 40,000 patients who stayed in critical care units of the Beth Israel Deaconess Medical Center between 2001 and 2012. The Dataset has 26 tables which includes vital signs, medications, laboratory measurements, observations and notes charted by care providers, fluid balance, procedure codes, diagnostic codes, imaging reports, hospital length of stay, survival data, and more. We downloaded the MIMIC-III demo dataset that is limited to 100 patients. While the number of patients were limited, the metadata and dataschema were identical [24, 25].

The evaluation process consisted of the following three steps: CDE extraction, CDE integration, and construction of semantic relationships among the CDEs. We counted the numbers of CDEs generated in each step as an evaluation measure of the structural efficiency for the 25 clinical documents and FHIR bulk sample data. However, the MIMIC-III demo dataset is provided as a relational database, containing tables of data relating to patients. A table is a data storage structure which is similar to a spreadsheet: each column contains consistent information (e.g., patient identifiers), and each row contains an instantiation of that information (e.g. a row could contain the integer 340 in the patient identifier column which would imply that the row's patient identifier is 340) [25]. We manually reviewed the relationships among the columns of each table, whether there were cases which were covered by our proposed CDE relationships and constraints.

\section{Results}

Overview of all types of semantic relationships

To address the semantic challenges described above, we defined atomic and composite CDEs using newly proposed three semantic types, i.e., hybrid, dictionary, and repeated, and three constraints, i.e., ordered, operated, and required, in addition to the existing two semantic relationship constraints, i.e., dependent and variable relationships, defined in our previous study. The newly defined composite semantic type replaced the old composite relationship constraint that we defined previously [10].

Figure 1 depicts atomic and composite CDEs along with their specific relationships and constraints. An aCDE can be constrained using variable and hybrid relationships by classifying them as variable and hybrid aCDEs, respectively. The definition of $\mathrm{CCDE}$ as a set of interrelated aCDEs in our previous study [10] was extended to include a clear definition, a separate identifier for reuse, and constraints among aCDEs included in a cCDE. A cCDEs can be classified into dictionary and repeated $\mathrm{CCDEs}$. The dependent relationship was the only relationship constraint in our previous study. We extended it to four constraints: ordered, operated, required, and dependent. As shown in the left lower panel in Fig. 1 , the ordered constraint does not apply to an aCDE.

\section{Data entries with multiple data types: Hybrid aCDE}

A hybrid aCDE is a particular type of aCDE that allows a value domain with multiple (or hybrid) data types. Technically it includes several aCDEs having the same $\mathrm{CDE}$ concept but different value domains. Figure $2 \mathrm{a}$ shows a part of a hemodialysis CRF from the DialysisNet and Avatar Beans Project. A time-tagged hybrid aCDE was applied to the Time attribute in a tabular data-entry format (Fig. 2a). Time is defined as a hybrid aCDE, Hemodialysis_Time_Hybrid_DE (DE:47616). Time is derived from two aCDEs, i.e., Hemodialysis_Time_DE (DE: 43239) and Hemodialysis_Time_String_DE (DE:47614) allowing a 'time' such as '08:00' and an 'enumeratedstring' such as 'Finish' and 'Start', data types, respectively (Fig. 2b). The hybrid aCDE Time (or Hemodialysis_ Time_Hybrid_DE (DE:47616)) can capture either a time or an enumerated string value as input.

\section{Tabular data entries: Repeated CCDE}

A repeated $\mathrm{CCDE}$ is a $\mathrm{CCDE}$ that captures data input multiple times in a tabular format. The definition of the repeated $\mathrm{CCDE}$ prevents the unnecessary creation of redundant CDEs and capture input data in a tabular format. A repeated $\mathrm{cCDE}$ efficiently captures and displays changes in input values over a certain time span, as shown in Fig. 2a. We first grouped eight aCDEs (i.e., DE: 47616, DE:43340, DE:43197, DE:43195, DE:43155, DE: 
A

\begin{tabular}{|c|c|c|c|c|c|c|c|}
\hline \multicolumn{8}{|c|}{ HEMODIALYSIS } \\
\hline Time & $\begin{array}{l}\text { Target Loss } \\
(\mathrm{kg})\end{array}$ & $\begin{array}{l}\text { Dialyate } \\
\text { Temp }\left({ }^{\circ} \mathrm{C}\right)\end{array}$ & $\begin{array}{l}\text { Dialyate Flow } \\
(\mathrm{ml} / \mathrm{min})\end{array}$ & $\begin{array}{l}\text { Blood Flow } \\
(\mathrm{ml} / \mathrm{min})\end{array}$ & A.P $(\mathrm{mmHg})$ & V.P $(\mathrm{mmHg})$ & B.P $(\mathrm{mmHg})$ \\
\hline 08.00 & 0.6 & 36.5 & 500 & 200 & -70 & 60 & $165 / 70$ \\
\hline $08: 30$ & 0.6 & 36.5 & 500 & 200 & -70 & 60 & $172 / 75$ \\
\hline 09.00 & 0.6 & 36.5 & 500 & 200 & .60 & 50 & $156 / 72$ \\
\hline $10-00$ & 0.6 & 36.5 & 500 & 200 & -70 & 60 & $158 / 73$ \\
\hline 1100 & 0.6 & 36.5 & 500 & 200 & -60 & 60 & $168 / 73$ \\
\hline $12: 00$ & 0.6 & 36.5 & 500 & 200 & -60 & 60 & $171 / 77$ \\
\hline FINISH & & & & & & & \\
\hline
\end{tabular}

B

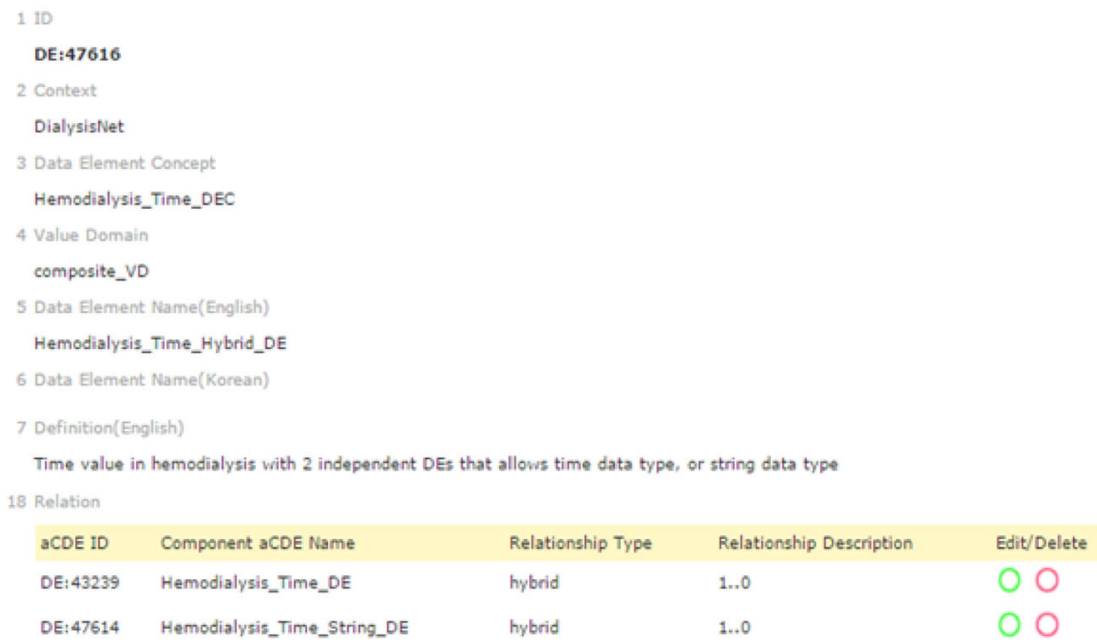

Fig. 2 An example hybrid aCDE from a hemodialysis report. a The hemodialysis table of the DialysisNet Project has a tabular data-entry format, where Time (DE:47616) allows two different data types: time and an enumerated string. b The hybrid aCDE (DE:47616) contains two aCDEs (DE:43239 and DE:47614) in a hybrid relationship (http://chmr2.snubi.org:8083/chmr/data_element_view.jsp?id=28476)

43092, DE:43372, and DE:43166) to create a cCDE and then assigned repeated relationship to create a repeated cCDE, Hemodialysis_Repeated_Componsite _DE (DE: 47575) (Fig. 3). As shown in Fig. 2, DE:47616 is a hybrid aCDE contained in a repeated $\mathrm{CCDE}$ (DE:47575).

\section{Dictionary data entries: Dictionary CCDE}

Our previous study [10] defined a variable CDE as a $\mathrm{CDE}$ that contains a controlled biomedical vocabulary variable. Similarly, a cCDE containing a variable aCDE as the primary key of a dictionary table can be defined as a dictionary $\mathrm{CCDE}$. This approach provides a way to encode an entire dictionary table as well as a controlled vocabulary into a single dictionary $\mathrm{cCDE}$, and thereby capture comprehensive biomedical knowledge from a database. A dictionary cCDE provides a useful means to apply relevant attributes of a dictionary database to constrain and validate input values to the dictionary $\mathrm{CCDE}$.

Figure 4a displays a typical data-entry document for laboratory test results in a tabular format. The 'Electrolyte Laboratory Tests' form from 'Recommended Labs for Stroke' of the NINDS CDE project [26] consists of six attributes including the laboratory test name, laboratory test result, unit of the laboratory test result, an indicator for whether the laboratory test result was abnormal, and another indicator for whether the laboratory test result was clinically significant when the laboratory test result was abnormal. Figure $4 \mathrm{~b}$ shows a part of the structured NINDS 'Electrolyte Laboratory Tests Dictionary' reference table. The Unit of Result attribute supports multiple units that are delimited by ' $\wedge$ '. The Normal Range attribute is also separated according to the Unit of Result and is represented in JSON (Javascript object notation)-type encoding.

A dictionary $\mathrm{cCDE}$ can systematically capture the entire 'Electrolyte Laboratory Tests' data-entry document as 'DE: 47571 Laboratory_Test_NINDS_Composite_DE,' which is composed of six aCDEs (Fig. 4c, Relation) that include a variable aCDE, 'DE:43938 Laboratory_Finding_Test_Name_DE,' which functions as the foreign key to refer to the primary key for the 'Lab Test Name' of the 'Electrolyte Laboratory Tests Dictionary' table of Fig. $4 \mathrm{~b}$. 


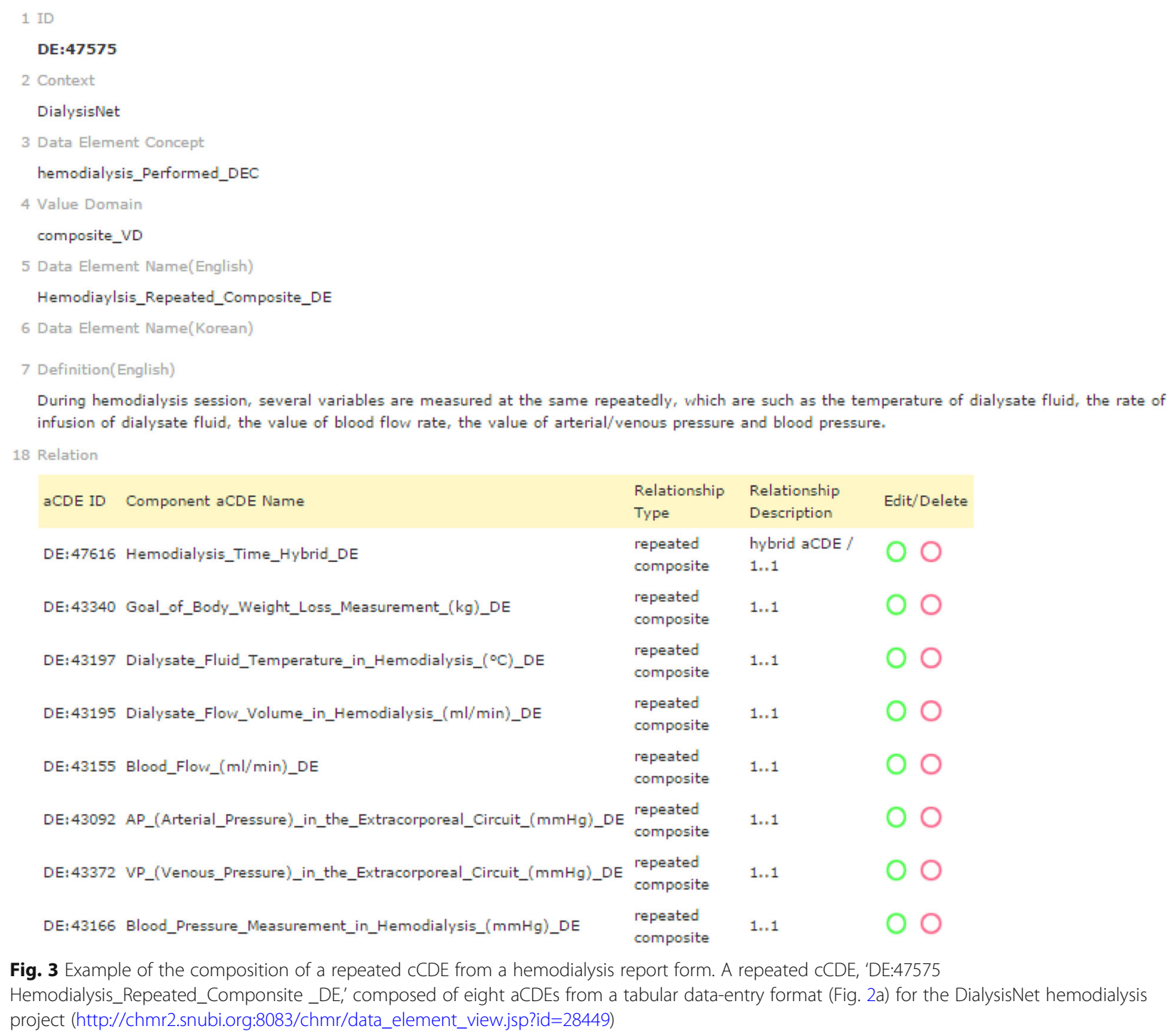

Fig. 3 Example of the composition of a repeated cCDE from a hemodialysis report form. A repeated cCDE, 'DE:47575 Hemodialysis_Repeated_Componsite_DE,' composed of eight aCDEs from a tabular data-entry format (Fig. 2a) for the DialysisNet hemodialysis project (http://chmr2.snubi.org:8083/chmr/data_element_view.jsp?id=28449)

Now that the dictionary cCDE, DE:47571, is successfully related to the NINDS 'Electrolyte Laboratory Tests' table via the variable aCDE (DE:43938), it provides a method to evaluate the validity of input value of $138 \mathrm{mEq} / \mathrm{L}$ to Result and Units for Result for a Test ['Sodium $\left.(\mathrm{Na}+)^{\prime}\right]$, with respect to the Normal Range (i.e., $135 \sim 145 \mathrm{mEq} / \mathrm{L}$ ) provided by the dictionary table connected via the primary key. The input value to Was test result abnormal? can also be automatically evaluated using the biomedical knowledge provided by the dictionary table. Moreover, when the value of Was test result abnormal? (DE:47566) is 'Abnormal,' the value of If abnormal, Clinically Significant? (DE:44135) can automatically be constrained to contain a value other than null. This constraint can be encoded by a Dependent Rule, as shown Relation Rule in Fig. 4c.

Figure 4c Relation Rule shows how a dictionary cCDE accompanied by its constraint rules are defined. For the two evaluation cases listed in Fig. 4b, both a Dictionary Rule and a Dependent Rule are defined by symbolic logic (or pseudocode) with the accompanying Descriptions. Dictionary Rule defines how to use biomedical knowledge contained in a dictionary table and Dependent Rule defines the interrelatedness of aCDEs in a cCDE by using dependent constraint relationship

\section{Semantic restriction: Constraints}

We defined four constraints that support the creation of a robust clinical document by specifying the interrelationship among many aCDEs. We defined four classes of operators: assignment, arithmetic, logical, and relational. Order can only be applied to aCDEs contained in a cCDE. However, the other three constraints (operated, required, and dependent) can be applied to both independent aCDEs and those contained in cCDEs (Fig. 1). We created 


\section{A Electrolyte Laboratory Tests}

\begin{tabular}{|l|l|l|l|l|l|}
\hline $\begin{array}{c}\text { Date Collected } \\
\text { (MM/DDMYY): }\end{array}$ & \multicolumn{1}{|c|}{ Test } & Result & Units for Result & $\begin{array}{l}\text { Was test result } \\
\text { abnormal? }\end{array}$ & $\begin{array}{l}\text { If abnormal, } \\
\text { Clinically } \\
\text { Significant? }\end{array}$ \\
\hline $01 / 08 / 2016$ & Sodium $\left(\mathrm{Na}^{+}\right)$ & 138 & $\mathrm{mEq} / \mathrm{L}$ & $\begin{array}{l}\square \text { Normal } \\
\square \text { Abnormal } \\
\square \text { Unknown }\end{array}$ & $\begin{array}{l}\square \text { Clinically } \\
\text { significant } \\
\text { a Not clinically } \\
\text { significant }\end{array}$ \\
\hline $01 / 08 / 2016$ & $\begin{array}{l}\text { Potassium } \\
(\mathrm{K}+)\end{array}$ & 5.3 & $\mathrm{mEq} / \mathrm{L}$ & $\begin{array}{l}\square \text { Normal } \\
\square \text { Abnormal } \\
\square \text { Unknown }\end{array}$ & $\begin{array}{l}\text { Clinically } \\
\text { significant } \\
\text { Not clinically } \\
\text { significant }\end{array}$ \\
\hline
\end{tabular}

\begin{tabular}{|c|c|c|c|c|c|c|}
\hline ID & Lab Class & Lab Test Name & Lab Test Definition & Data Type & Unit of Result & Normal Range \\
\hline 1 & $\begin{array}{l}\text { Electrolyte } \\
\text { Laboratory } \\
\text { Test }\end{array}$ & Sodium $(\mathrm{Na}+)$ & $\begin{array}{l}\text { A measurement of } \\
\text { the sodium ( } \mathrm{Na}+\text { ) in } \\
\text { a biological } \\
\text { specimen. SI or } \\
\text { American Units are } \\
\text { preferred. }\end{array}$ & Numeric & $\mathrm{mg} / \mathrm{dL}^{\wedge} \mathrm{mEq} / \mathrm{L}$ & $\begin{array}{l}\text { [र } \\
\text { "unit": "mg/dL", } \\
\text { "range": "31-34" } \\
\text { \}, \{ } \\
\text { "unit": "mEq/L", } \\
\text { "range": "135-145 }\end{array}$ \\
\hline 2 & $\begin{array}{l}\text { Electrolyte } \\
\text { Laboratory } \\
\text { Test }\end{array}$ & Potassium (K+) & $\begin{array}{l}\text { A measurement of } \\
\text { the potassium }(\mathrm{K}+) \\
\text { in a biological } \\
\text { specimen. SI or } \\
\text { American Units are } \\
\text { preferred. }\end{array}$ & Numeric & $\mathrm{mg} / \mathrm{dL} \wedge \mathrm{mEq} / \mathrm{L}$ & 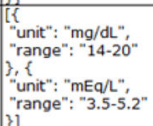 \\
\hline
\end{tabular}

C

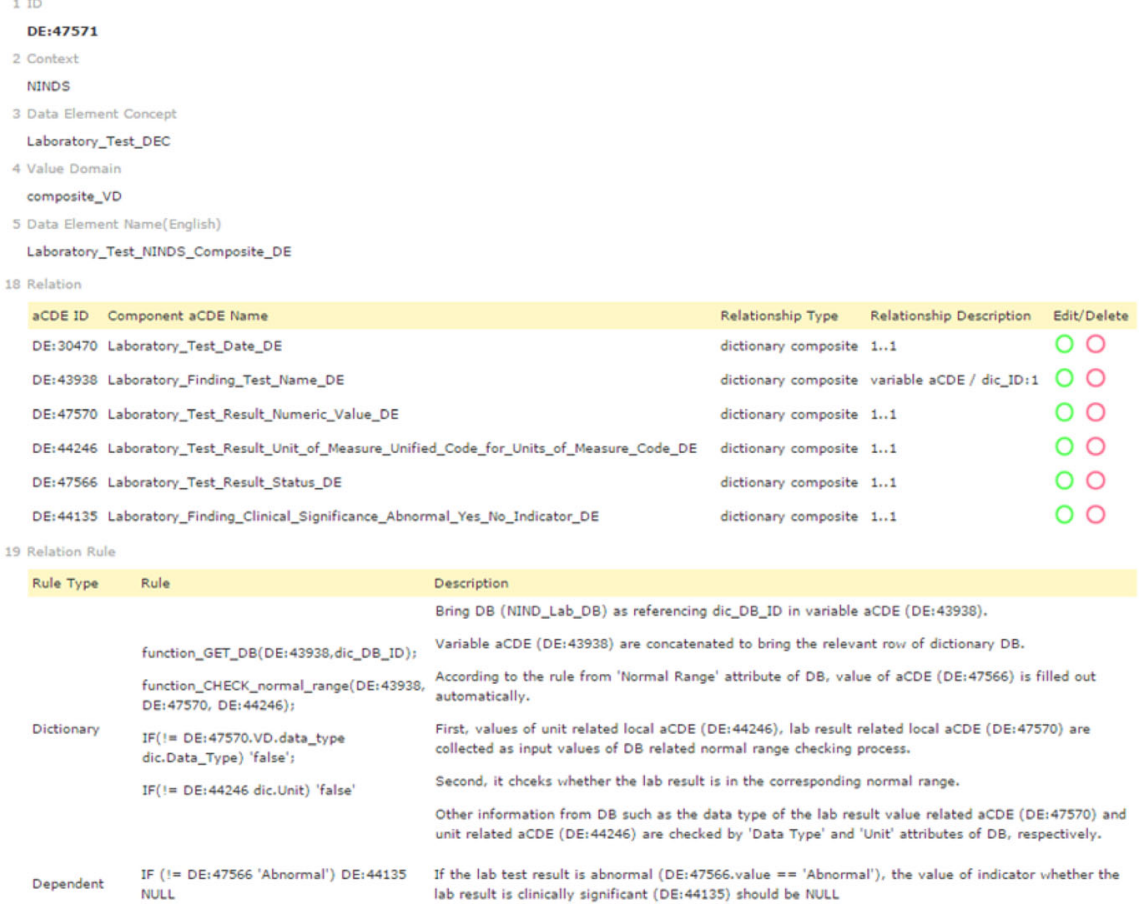

Fig. 4 Creation of a dictionary CCDE for a CRF. a The 'Electrolyte Laboratory Tests' table on a clinical document is provided as an example tabular dataentry document to capture laboratory test results for sodium ( $\mathrm{Na}+$ ) and potassium $(\mathrm{K}+$ ) along with two clinical evaluation attributes. b We constructed the 'Electrolyte Laboratory Tests Dictionary' table by extracting the relevant attributes from the CDEs defined in the 'Recommended Labs for Stroke' from the NINDS CDE project. c The dictionary CCDE (DE:47571) consists of six aCDEs that include a variable aCDE (DE:43938) that relates the dictionary cCDE to the dictionary table in Fig. 4b. Two rules for clinical evaluation are presented (http://chmr2.snubi.org:8083/chmr/data_element_view.jsp?id=28445)

symbolic logic with prefix notation [27] (Table 1) to describe the order of operations and to formulate constraints. More practical examples are shown in Fig. 5 to demonstrate how constraints are applied to a repeated cCDE as well. The four constraints are described as follows:

1) Operated. Table $1 \mathrm{~A}$ presents the standard BMI formula [BMI (in $\mathrm{kg} / \mathrm{m}^{2}$ ) $=$ weight $/$ (height $x$ height)] in a prefix notation as ( / CDE30 CDE31 CDE31 100,100), where CDE30 and CDE31 represent Body Weight Value in kg and Body Height Value in $\mathrm{cm}$, respectively. Both ' $\mathrm{cm}$ ' and 'm' units of height measurements are supported by IF conditional statement to manage different units: ( IF ( = CDE31.unit_of_measure ' $\mathrm{m}$ ') ( / CDE30 CDE31 CDE31) (/ CDE30 CDE31 CDE31 $100,100))$. 
Table 1 Encoding operated, required, dependent, and ordered constraints for CDEs with prefix notation. Examples of (A) an operated constraint for calculating BMI, (B) a required constraint for demography information, (C) a dependent constraint for smoking history, and (D) an ordered constraint

\begin{tabular}{|c|c|c|}
\hline \multirow[t]{2}{*}{ Constraints } & Example of Clinical Documents & \multirow[t]{2}{*}{ Set of CDE IDs and Names } \\
\hline & Prefix Notation for Formulating Constraints & \\
\hline \multirow[t]{2}{*}{ A) Operated } & $\begin{array}{l}\text { Weight }(\mathrm{kg}) \text { : } \\
\text { Height }(\mathrm{cm}) \text { : } \\
\text { BMI }\left(\mathrm{kg} / \mathrm{m}^{2}\right) \text { : }\end{array}$ & \multirow[t]{2}{*}{$\begin{array}{l}\text { CDE30 Body Weight Value in } \mathrm{kg} \\
\text { CDE31 Body Height Value in } \mathrm{cm} \\
\text { CDE32 Body Mass Index Value }\end{array}$} \\
\hline & $\begin{array}{l}\text { (IF ( = CDE31.unit_of_measure 'm') } \\
(/ \mathrm{CDE} 30 \mathrm{CDE} 31 \mathrm{CDE} 31)(/ \mathrm{CDE} 30 \mathrm{CDE} 31 \\
\mathrm{CDE} 31100,100)) \\
(/ \mathrm{CDE} 30 \mathrm{CDE} 31 \mathrm{CDE} 31100,100)\end{array}$ & \\
\hline \multirow[t]{2}{*}{ B) Required } & $\begin{array}{l}\text { 1) * Patient Age: } \\
\text { 2) *Gender } \\
\text { Female Male Unknown } \\
\text { Unspecified Not reported } \\
\text { 3) Ethnicity: } \\
\text { Hispanic or Latino Unknown } \\
\text { Not Hispanic or Latino Not reported }\end{array}$ & \multirow[t]{2}{*}{$\begin{array}{l}\text { CDE40 Patient Age } \\
\text { CDE41 Patient Gender } \\
\text { CDE42 Patient Ethnicity }\end{array}$} \\
\hline & (Required CDE 40 CDE 41 ) & \\
\hline \multirow[t]{2}{*}{ C) Dependent } & $\begin{array}{l}\text { Smoking History } \\
\text { 1) *Current tobacco use? } \\
\text { Yes No Unknown } \\
\text { 2) *Past tobacco use? } \\
\text { Yes No Unknown } \\
\text { 3) Age when tobacco use started (years)? } \\
\text { (Skip if Q1 and Q2 are both No) }\end{array}$ & \multirow[t]{3}{*}{$\begin{array}{l}\text { CDE20 Current Smoking Indicator } \\
\text { CDE21 Past Smoking Indicator } \\
\text { CDE22 Age When Tobacco Use Started }\end{array}$} \\
\hline & $\begin{array}{l}(\text { IF (or }(!=\text { CDE20 'Yes') }(!=\operatorname{CDE} 21 \\
\text { 'Yes' }) \text { ) CDE22 NULL) }\end{array}$ & \\
\hline D) Ordered & (Ordered CDE20 CDE21 CDE22) & \\
\hline
\end{tabular}

2) Required. A Required constraint applied to an aCDE means that the aCDE must have a value other than null. Table $1 \mathrm{~B}$ lists the demographic information of a clinical document, constraining "Patient Age (CDE40)' and '"Gender (CDE41)' as required by the statement (Required CDE $40 \mathrm{CDE} 41$ ).

3) Dependent. It might be necessary to dynamically enable or disable a certain aCDE according to the value(s) of other aCDE(s). For example, a genderspecific CDE might only be applied to subjects of the applicable gender. Table $1 \mathrm{C}$ presents an example for checking whether a patient is a current $(C D E 20)$ or past (CDE21) smoker in order to obtain the age when tobacco use was started (CDE22). A nonsmoker can conveniently skip CDE22 if ( = CDE2 0 CDE2 1 'No') by setting the value of CDE22 as null. In other words, a rule such as (IF ( or $(!=\operatorname{CDE} 20$ 'Yes') $(!=\operatorname{CDE} 21$ 'Yes') ) CDE22 NULL) can be imposed. Another constraint can be imposed to check illogical input values such as ( = CDE20 CDE21 'Yes') if necessary.

4) Ordered. The ordering of aCDEs (especially in a cCDE) is important for certain conditions and contexts. CDEs in Table $1 \mathrm{C}$ can be ordered by a constraint statement such as (Ordered CDE2 0 CDE21 CDE22).

\section{Evaluation study}

To evaluate the usefulness of our newly extended composite semantic relationships, we applied them to CDEs which were systematically extracted from the 25 clinical documents of five teaching hospitals in Korea and from FHIR bulk sample data. At first, we focused on deriving CDEs from clinical documents, which provided many explicit cases that clearly demonstrated the relationships between CDEs. We then wanted to prove that our proposed relationships and constraints were valid in structured clinical dataset as well. It was why we chose two difference types of source data: unstructured and structured data. The evaluation process consisted of the following steps: CDE extraction, $\mathrm{CDE}$ integration by using the newly proposed atomic and composite CDEs with semantic enrichments. We examined how the number of CDEs had been reduced from $\mathrm{CDE}$ extraction to $\mathrm{CDE}$ integration, measuring the structural and semantic efficiency of CDEs for clinical data elements.

Although HL7 FHIR supports mainly structured data, it also provides a document related resource, FHIR Questionnaire. To see whether our proposed semantic types can cover FHIR Questionnaire, we matched elements of the FHIR Questionnaire resource to our developed relationships and constraints for further evaluation.

For evaluating derived CDEs from clinical documents, we first extracted $84,48,70,83$, and $37 \mathrm{CDEs}$ from the 


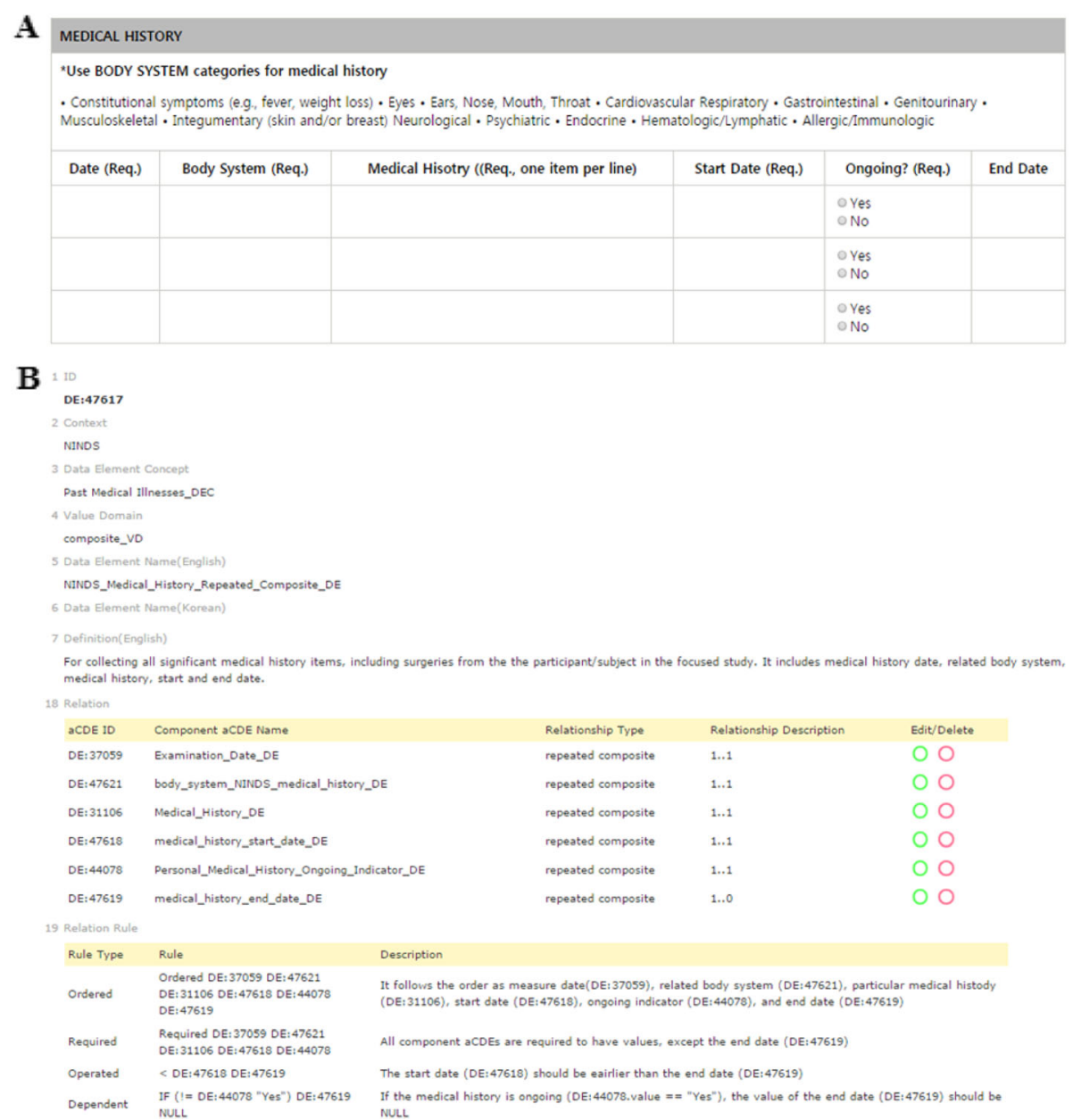

Fig. 5 Encoding Operated, Ordered, Required, and Dependent constraints for a repeated CCDE. a A 'Medical History' clinical document presented in a tabular format containing six attributes. b A repeated CCDE is created with the corresponding six aCDEs along with four constraint rules: (1) the start date (DE:47618) should be earlier than the end date (DE:47619): (<DE:47618 DE:47619); (2) all attributes are required to have values other than null, except for the end date (DE:47619): (Required DE: $37059 \mathrm{DE}: 47621 \mathrm{DE}: 31106 \mathrm{DE}: 47618 \mathrm{DE}: 44078,3)$ when a certain medical history is not ongoing (DE:44078), the end date (DE:47619) cannot be obtained, and vice versa: (IF ( ! = DE : 44078 'Yes' ) $\mathrm{DE}: 47619$ NULL); and (4) aCDEs can be ordered according to a constraint statement such as (Ordered DE : $37059 \mathrm{DE}: 47621 \mathrm{DE}: 31106$ $\mathrm{DE}: 47618 \mathrm{DE}: 44078 \mathrm{DE}: 47619)$ (http://chmr2.snubi.org:8083/chmr/data_element_view.jsp?id=28477)

five clinical documents, Admission Note, Initial Medical Examination, Discharge Summary, Emergency Note, and Operation Note, respectively, of Hospital A. We found that $95(29.5 \%)$ of the 322 aCDEs were reused in at least two of the five clinical documents, resulting in 227 unique aCDEs. We then created clinically relevant cCDEs and applied semantic relationships to them. Of the 84 aCDEs extracted from the Admission Note of Hospital A, 55 were successfully captured by 10 cCDEs. Finally, 16 cCDEs successfully captured 110 (48.5\%) of the 227 unique CDEs such that $133(=16+117)$ CDEs $(41.3 \%)$ were sufficient to represent the initial $322 \mathrm{CDEs}$ extracted from the five clinical documents of Hospital A (Table 2).

In the CDE extraction step, we found that applying $\mathrm{CDE}$ is an effective way to reduce redundant CDEs (22.2 $37.9 \%)$ at each hospital. This means that there were many
CDEs shared across the five different clinical documents at each hospital. We found that an even higher CDE reduction rate of $48.7 \%$ was achieved by integrating the information for all five hospitals, which indicated that various CDEs were commonly used across five different teaching hospitals.

The CDE integration step involved integrating aCDEs into clinically relevant $\mathrm{CCDEs}$ to further structure the clinical documents and then integrating the cCDEs across different clinical documents. For example, when a vital sign-related CCDE contained three aCDEs ('body weight,' 'body temperature,' and 'blood pressure') and another vital sign-related $\mathrm{CCDE}$ contained an additional aCDE ('description the reason of unstable vital sign'), we integrated them into a vital-sign cCDE comprising four aCDEs. The application of these three steps constantly 
Table 2 Numbers of aCDEs and CCDEs extracted from five clinical documents used at five teaching hospitals in Korea

\begin{tabular}{|c|c|c|c|c|c|c|c|c|c|}
\hline Hospital & & $\begin{array}{l}\text { Admission } \\
\text { Note }\end{array}$ & $\begin{array}{l}\text { Initial Medical } \\
\text { Examination } \\
\text { Note }\end{array}$ & $\begin{array}{l}\text { Discharge } \\
\text { Summary }\end{array}$ & $\begin{array}{l}\text { Emergency } \\
\text { Not }\end{array}$ & $\begin{array}{l}\text { Operation } \\
\text { Note }\end{array}$ & $\begin{array}{l}\text { Total No. of } \\
\text { CDEs }\end{array}$ & $\begin{array}{l}{ }^{f} \text { No. of Unique } \\
\text { CDEs }\end{array}$ & $\begin{array}{l}{ }^{9} \text { CDE Reuse } \\
\text { Rate }\end{array}$ \\
\hline \multirow[t]{4}{*}{ A } & ${ }^{\mathrm{a}} \mathrm{CDE}$ & 84 & 48 & 70 & 83 & 37 & 322 & 227 & $29.5 \%$ \\
\hline & $\begin{array}{l}{ }^{b}{ }_{C C D E}{ }^{c} \\
(a C D E)\end{array}$ & $10(55)$ & $9(40)$ & $6(34)$ & $6(45)$ & $2(10)$ & $33(184)$ & $16(110)$ & \\
\hline & ${ }^{d} \mathrm{aCDE}$ & 29 & 8 & 36 & 38 & 27 & 138 & 117 & \\
\hline & $\begin{array}{l}{ }^{e} \mathrm{CCDE}+ \\
\mathrm{aCDE}\end{array}$ & 39 & 17 & 42 & 44 & 29 & 171 & 133 & $24.5 \%$ \\
\hline \multirow[t]{4}{*}{ C } & CDE & 30 & 35 & 20 & 27 & 26 & 138 & 87 & $37.0 \%$ \\
\hline & $\begin{array}{l}\mathrm{cCDE} \\
(\mathrm{aCDE})\end{array}$ & $2(14)$ & $3(20)$ & $2(11)$ & $3(15)$ & $1(5)$ & $11(65)$ & $5(35)$ & \\
\hline & $\mathrm{aCDE}$ & 16 & 15 & 9 & 12 & 21 & 73 & 52 & \\
\hline & $\begin{array}{l}\text { cCDE + } \\
\text { aCDE }\end{array}$ & 18 & 18 & 11 & 15 & 22 & 84 & 57 & $33.3 \%$ \\
\hline \multirow[t]{4}{*}{ G } & CDE & 70 & 28 & 44 & 54 & 11 & 207 & 161 & $22.2 \%$ \\
\hline & $\begin{array}{l}\mathrm{cCDE} \\
(\mathrm{aCDE})\end{array}$ & $4(23)$ & $3(17)$ & $2(11)$ & $2(17)$ & $1(5)$ & $12(73)$ & $7(50)$ & \\
\hline & $\mathrm{aCDE}$ & 47 & 11 & 33 & 37 & 6 & 134 & 111 & \\
\hline & $\begin{array}{l}\text { CCDE + } \\
\mathrm{aCDE}\end{array}$ & 51 & 14 & 35 & 39 & 7 & 146 & 118 & $18.8 \%$ \\
\hline \multirow[t]{4}{*}{$P$} & CDE & 204 & 123 & 46 & 43 & 12 & 428 & 266 & $37.9 \%$ \\
\hline & $\begin{array}{l}\mathrm{cCDE} \\
\text { (aCDE) }\end{array}$ & 7 (177) & $4(99)$ & $3(34)$ & $3(39)$ & $0(0)$ & 15 (349) & $7(177)$ & \\
\hline & $\mathrm{aCDE}$ & 27 & 24 & 12 & 4 & 12 & 79 & 89 & \\
\hline & $\begin{array}{l}\text { CCDE + } \\
\text { aCDE }\end{array}$ & 34 & 28 & 15 & 7 & 12 & 94 & 96 & $36.2 \%$ \\
\hline \multirow[t]{4}{*}{ S } & CDE & 12 & 6 & 9 & 10 & 10 & 47 & 31 & $34.0 \%$ \\
\hline & $\begin{array}{l}\mathrm{cCDE} \\
(\mathrm{aCDE})\end{array}$ & $1(3)$ & 0 & 0 & $1(4)$ & 0 & $2(7)$ & $1(4)$ & \\
\hline & $\mathrm{aCDE}$ & 9 & 6 & 9 & 6 & 10 & 40 & 27 & \\
\hline & $\begin{array}{l}\text { CCDE + } \\
\text { aCDE }\end{array}$ & 10 & 6 & 9 & 7 & 10 & 42 & 28 & $31.9 \%$ \\
\hline \multirow[t]{5}{*}{ Total } & $\mathrm{CDE}$ & 400 & 240 & 189 & 217 & 96 & 1142 & 606 & $53.1 \%$ \\
\hline & Unique CDE & 297 & 162 & 142 & 178 & 57 & 836 & 586 & $29.9 \%$ \\
\hline & $\begin{array}{l}\mathrm{cCDE} \\
(\mathrm{aCDE})\end{array}$ & $15(224)$ & $14(152)$ & $9(71)$ & $9(90)$ & $2(10)$ & 49 (547) & $20(327)$ & \\
\hline & $\mathrm{aCDE}$ & 73 & 10 & 71 & 88 & 47 & 289 & 259 & \\
\hline & $\begin{array}{l}\text { CCDE + } \\
\mathrm{aCDE}\end{array}$ & 88 & 24 & 80 & 97 & 49 & 338 & 279 & $46.9 \%$ \\
\hline
\end{tabular}

\footnotetext{
${ }^{a}$ No. of CDEs extracted from each clinical document from each hospital

${ }^{b}$ No. of cCDEs created for each clinical document

${ }^{c}$ No. of aCDEs contained in ${ }^{b} \mathrm{cCDEs}$

${ }^{d}$ No. of remaining aCDEs that are not contained in any of the CCDEs in each clinical document

${ }^{e}$ Total no. of CDEs consisting of ${ }^{b} \mathrm{cCDEs}$ and ${ }^{\mathrm{d}} \mathrm{aCDEs}$ that are not contained in any of the cCDEs in each clinical document

${ }^{f}$ No. of unique CDEs across the five clinical documents

${ }^{\mathrm{g}}$ Reuse ratio of CDEs across the five clinical documents
}

decreased the number of CDEs. Supplementary Tables S3-S5 list the CCDEs and how they were distributed in each document at each hospital. These tables also provide a detailed view of how the 20 unique cCDEs comprised 327 sub-aCDEs. The integrated CDEs not only reduced the number of CDEs with a reuse ratio of up to
$46.9 \%[=(1142-20-586) / 1142]$ (Table 2) but also showed greatly improved semantic accuracy and interoperability, which was also supported by the review of the documents by the authors.

We found that the compositions of the clinical documents differed quite markedly across the five hospitals. 
The clinical documents at Hospitals $\mathrm{P}$ and $\mathrm{S}$ contained the largest $(n=266)$ and smallest $(n=31)$ numbers of independent CDEs, respectively. We also found that even the same clinical documents showed huge variations in CDE numbers. The number of CDEs in Admission Notes varied from 12 at Hospital S to 204 at Hospital P. Hospital $\mathrm{P}$ also had the largest number of aCDEs for Initial Medical Examination Note $(n=123)$ while Hospital A had the largest number of aCDEs for Emergency Note $(n=83)$ and Operation Note $(n=37)$.

We also applied constraint rules for the five clinical documents of the five hospitals (Table 3). We could not determine if a CDE was a hybrid aCDE partly due to the lack of sufficient input values and partly due to poor descriptions of the response values for the clinical documents. We designated the cCDEs as basic cCDEs to distinguish them from repeated and dictionary cCDEs. A cCDE was on average reused twice among the five documents by the hospitals. We also found that the clinical documents at Hospital A were the best structured and contained the greatest detail with more $\mathrm{cCDEs}$ and constraint rules compared to the documents of the other hospitals.

We evaluated the DE relationships and constraints with the same method applied to different data sources, which were 14 FHIR resources from FHIR bulk sample data. We first extracted $238 \mathrm{CDEs}$ and found $142 \mathrm{CDEs}$ (59.7\%) were reused in at least 2 of 14 FHIR resources, resulting in 96 unique aCDEs. We then created clinically relevant cCDEs and applied semantic relationships to them. 48 cCDEs successfully captured 194 (81.5\%) of 238 CDEs. Finally, 28 cCDEs successfully captured 75 of the 96 unique CDEs such that $49(=28+21)$ CDEs were enough to represent the initial 238 CDEs extracted from 14 FHIR resources (Table 4). Supplementary Tables S6S7 list the cCDEs and how they were distributed in each FHIR resources. The fact that more than half of the CDEs has been reused shows that the FHIR data are relatively well standardized and structured. Half of the

Table 3 Numbers of aCDEs, cCDEs, and constraints at five teaching hospitals in Korea

\begin{tabular}{lllllll}
\hline Hospital: CDE Semantic Type & $\mathrm{A}$ & $\mathrm{C}$ & $\mathrm{G}$ & $\mathrm{P}$ & $\mathrm{S}$ \\
\hline aCDE & Hybrid & 0 & 0 & 0 & 0 & 0 \\
& Variable & 5 & 2 & 2 & 3 & 0 \\
cCDE & Basic & $9(20)$ & $2(6)$ & $3(8)$ & $2(2)$ & 0 \\
& Repeated & $2(5)$ & $1(2)$ & $2(2)$ & $2(6)$ & $1(2)$ \\
& Dictionary & $5(10)$ & $2(3)$ & $2(2)$ & $3(8)$ & 0 \\
\multirow{4}{*}{ Constraints } & Operated & $4(9)$ & $1(5)$ & $2(5)$ & $1(1)$ & 0 \\
& Required & $10(25)$ & $3(8)$ & $5(11)$ & $3(11)$ & 0 \\
& Dependent & $15(26)$ & 0 & $3(8)$ & $3(10)$ & $1(2)$ \\
& Ordered & $11(29)$ & $4(10)$ & $5(11)$ & $3(12)$ & $1(2)$ \\
\hline
\end{tabular}

The numbers before the parentheses represent unique counts
FHIR resources, i.e., AllergyIntolerance, Condition, Encounter, Goal, MedicationRequest, Organization, and Procedure, were represented by repeated cCDEs, which means all extracted CDEs of each FHIR resource became a component aCDEs of the repeated cCDEs. These structured data have been reused frequently among different FHIR resources.

While we were mapping our proposed semantic types and constraints to FHIR resources, we found that hybrid $\mathrm{aCDE}$ and operated, and dependent constraints were not applicable in FHIR resources. For the case of hybrid aCDE, although only one datatype is allowed for each data in FHIR specification, we foun no restriction on the datatype in the FHIR bulk sample data since the data was represented by JSON, and XML. While the required and ordered constraints were explicitly indicated, operated, and dependent constraints were not valid in FHIR resources because the rule by which two or more data values were related could not be applied (Table 5).

Another evaluation was the mapping between our semantic types and constraints to document-associated FHIR resource, Questionnaire. Figure 6 represents the mapping of the FHIR structure in extracts on the left side, linked via arrows to the corresponding developed CDE relationships and constraints. The relevant elements in the FHIR Questionnaire resource were group and question, which represents composite and atomic CDEs (the data model of a single question). Among our three CDE relationships and four constraints, the repeated $\mathrm{CCDE}$ relationship and the required and operated constraints were straightforwardly mapped. The FHIR Questionnaire resource is to define both collection forms, surveys and other structures that can be filled out with their context. It had a certain structure to represent relationships among CDEs but value related constraints could not be modelled. For instance, it could not be represented whether the value allows for multiple data types (Hybrid aCDE) or whether one value can be changed depending upon another element's value (Constraint: Dependent).

For evaluations with a real dataset, we analyzed 26 tables of the MIMIC-III demo database. These tables were divided into three categories which were classified by different data characteristics: (1) 14 tables for hospital data, (2) three tables for online definitions, and (3) 19 tables for care-value and meta-version ICU related data (Supplementary Tables S8). We first manually reviewed the relationships among the columns of each table. The evaluation process was conducted only for cases in which a relationship was found through the following steps: CDE extraction, CDE integration by using atomic and composite CDEs and then the construction of semantic relationships among the CDEs.

We found four hybrid aCDEs that allows numeric data and text data. For example, VALUE in LABEVENTS 
Table 4 Numbers of atomic and composite CDEs extracted from 14 FHIR resources of FHIR bulk sample data

\begin{tabular}{|c|c|c|c|c|c|}
\hline$\#$ & FHIR Resource & ${ }^{\mathrm{a}} \mathrm{CDE}$ & ${ }^{b}{ }^{c} C^{\prime} E^{c}(a C D E)$ & ${ }^{d}$ aCDE & ${ }^{\mathrm{e}} \mathrm{cCDE}+\mathrm{aCDE}$ \\
\hline 1 & Allergylntolerance & 13 & $2(13)$ & 0 & 2 \\
\hline 2 & CarePlan & 18 & $4(15)$ & 3 & 7 \\
\hline 3 & Claim & 21 & $5(13)$ & 6 & 11 \\
\hline 4 & Condition & 13 & $2(13)$ & 0 & 2 \\
\hline 5 & DiagnosticReport & 13 & $3(9)$ & 4 & 7 \\
\hline 6 & Encounter & 15 & $4(15)$ & 0 & 4 \\
\hline 7 & Goal & 4 & $1(4)$ & 0 & 1 \\
\hline 8 & ImagingStudy & 23 & $3(14)$ & 11 & 14 \\
\hline 9 & Immunization & 12 & $1(4)$ & 8 & 9 \\
\hline 10 & MedicationRequest & 14 & $3(14)$ & 0 & 3 \\
\hline 11 & Observation & 22 & $5(18)$ & 4 & 9 \\
\hline 12 & Organization & 15 & $4(15)$ & 0 & 4 \\
\hline 13 & Patient & 42 & $8(29)$ & 8 & 16 \\
\hline 14 & Procedure & 13 & $3(13)$ & 0 & 3 \\
\hline \multicolumn{2}{|c|}{${ }^{\mathrm{f}}$ Total No. of CDEs } & 238 & 48 (194) & 44 & 92 \\
\hline \multicolumn{2}{|c|}{${ }^{9}$ No. of unique CDEs } & 96 & $28(75)$ & 21 & 49 \\
\hline
\end{tabular}

${ }^{\mathrm{a}}$ No. of CDEs extracted from each FHIR resource sample data

${ }^{b}$ No. of CCDEs created for each FHIR resource sample data

${ }^{\mathrm{c}}$ No. of aCDEs contained in ${ }^{\mathrm{b}} \mathrm{CCDES}$

${ }^{d}$ No. of remaining aCDEs that are not contained in any of the CCDEs in each FHIR resource sample data

${ }^{e}$ Total no. of CDEs consisting of ${ }^{b} C C D E s$ and ${ }^{d} a C D E s$ that are not contained in any of the $C C D E s$ in each FHIR resource sample data

${ }^{f}$ Total no. of CDEs across 14 FHIR resources

${ }^{g}$ Total no. of unique CDEs across 14 FHIR resources

allows for string data and numeric data. If this value is numeric, then VALUENUM represents the same data in a numeric format with an appropriate unit from VALUEUOM for its usability in calculations. The four general cCDEs in Table 5 list cCDEs that includes the hybrid aCDE. We also found three variable aCDEs associated with its particular dictionary cCDE. For example, ICD9_CODE in DIAGNOSES_ICD is matched to the same value as ICD9_CODE in D_ICD_DIAGNOSES. And each table became a repeated $\mathrm{CCDE}$ because it is composed of a set of related items. All tables have a required constraint, and two tables have an operated

Table 5 Numbers of atomic and composite CDEs and constraints in FHIR bulk data and MIMIC-III demo data

\begin{tabular}{llll}
\hline Data Source: CDE Semantic Type & FHIR & MIMIC-III \\
\hline aCDE & Hybrid & N/A & 4 \\
& Variable & 3 & 4 \\
cCDE & General & $18(64)$ & $4(12)$ \\
& Repeated & $7(87)$ & $26(180)$ \\
& Dictionary & $3(17)$ & $4(17)$ \\
Constraints & Operated & N/A & 2 \\
& Required & 34 & 52 \\
& Dependent & N/A & N/A \\
& Ordered & 2 & N/A \\
\hline
\end{tabular}

constraint. As MIMIC data is provided as a relational database, dependent and/or ordered constraints are not applicable. Relational table treats the value of each column independently without ordering based on set inclusion theory (Table 5). Supplementary Tables S8-S9 lists specific results which the MIMIC-III metadata matched to our proposed relationships and constraints.

\section{Discussion}

\section{Comparison with related studies}

Standardization of clinical data using CDEs based on ISO/IEC 11179 metadata standard is clearly one of most effective ways to harmonize data collected from various clinical institutions and studies. The advantages of this approach are (1) providing a consistent data collection tool, (2) improving study data quality, and (3) reducing the cost of data entry and cleansing by having uniform data. However, the limitation of ISO/IEC 11179 of not providing a data structure for representing interrelationships among CDEs has resulted in a gap between the development of CDEs and their utilization in clinical forms. Although ISO/IEC 11179 provides DDEs to overcome the limitation by enhancing interrelated CDEs. A $\mathrm{DDE}$ is a DE whose values are derived through a transformation of the values of one or more source CDEs. For example, the DDE of the 'length of stay in a hospital' 


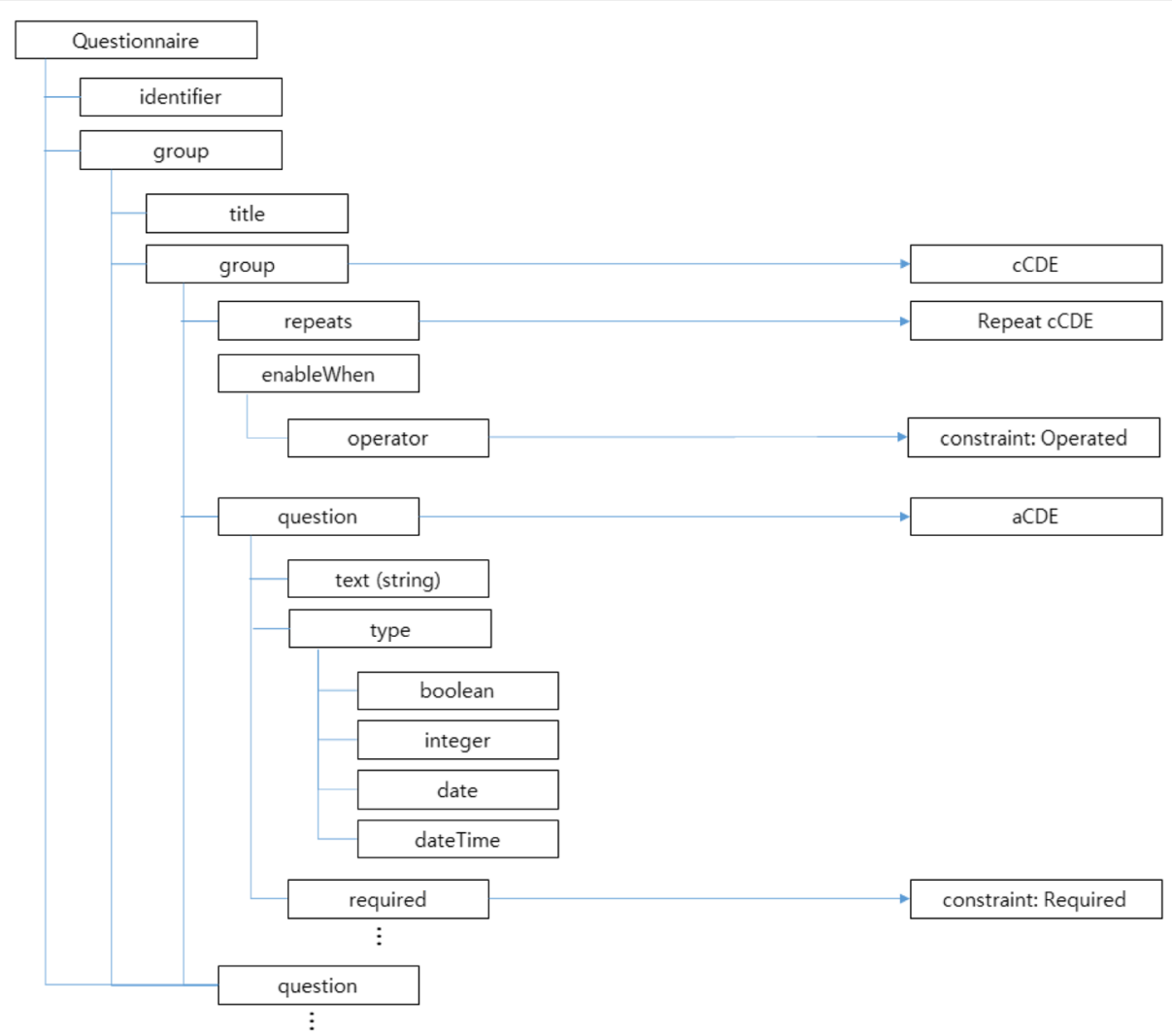

Fig. 6 Mapping result of the FHIR Questionnaire resource mapped to the proposed CDE relationships and constraints

is derived from two independent DEs that counts the number of days from two input CDEs: 'Admission date' and 'Discharge date.' However, this strategy is far from enough to cover all use cases of interrelated CDEs that we have describe in the Background section.
Table 6 compares the DDE and our CDE semantic relationships. The value of a DDE is derived from input $\mathrm{DE}(\mathrm{s})$. Our CDE semantic relationship provides rich semantics for creating atomic and composite CDEs that feature repeat and dictionary properties, supporting references to

Table 6 Differences between DDE and our CDE semantic relationships

\begin{tabular}{|c|c|c|c|}
\hline \multicolumn{2}{|c|}{ CDE Semantic Type } & \multirow{2}{*}{$\begin{array}{l}\text { Characteristic } \\
\text { Allowing the entry of multiple data types in a hybrid } \\
\text { aCDE requires aCDEs that support different data types } \\
\text { for the same data item }\end{array}$} & \multirow{2}{*}{$\begin{array}{l}\text { Difference from a DDE } \\
\text { A DDE does not support the entry of multiple types of data }\end{array}$} \\
\hline $\mathrm{aCDE}$ & Hybrid & & \\
\hline & Variable & Connecting to an outside dictionary database & No dictionary-associated constraint in a DDE \\
\hline \multirow[t]{3}{*}{ CCDE } & General & Containing a set of aCDEs & Do not have output DE(s), but a DDE can be a cCDE \\
\hline & Repeated & Allowing sequential data entry into a repeated $\mathrm{CCDE}$ & No repeated property in a DDE \\
\hline & Dictionary & $\begin{array}{l}\text { Bringing biomedical knowledge from an outside } \\
\text { dictionary database to a dictionary CCDE containing } \\
\text { a variable aCDE as a foreign key to the dictionary } \\
\text { table with the repeated property }\end{array}$ & No dictionary connection allowed for a DDE \\
\hline \multirow[t]{4}{*}{ Constraint } & Operated & $\begin{array}{l}\text { Allowing mathematical/algebraic expressions between } \\
\text { related aCDEs }\end{array}$ & A DDE has this constraint with the ${ }^{a}$ CALCULATION type \\
\hline & Required & Forcing aCDE to have a value other than null & No required constraint in a DDE \\
\hline & Dependent & Dynamic enabling and disabling of an aCDE via a predicate & No dependent constraint in a DDE \\
\hline & Ordered & Ordering a set of aCDEs & A DDE has this constraint by default \\
\hline
\end{tabular}

a CALCULATION type in DDE only covers arithmetic operators (i.e., $+,-,{ }^{*}, /$ ) but, the operated constraints include not only arithmetic operators but also logical operators (i.e., $<,>$ ) 
outside biomedical resources as described in Table 6 . The relatively simple-minded concept of the DDE may be insufficient to cover various CDE semantic relationships since a DDE covers only two constraints: Operated and Ordered.

There have also been efforts to address the issues of interrelated $\mathrm{CDE}(\mathrm{s})$ by applying external data models. The CDISC (clinical data interchange standards consortium) ODM (operational data model), which is an XMLbased standardized data model that supports the acquisition and exchange of metadata specifically related to clinical studies, can also be used to overcome the limitations of ISO/IEC 11179. However, it is not sufficiently comprehensive to generate CRFs by importing elements directly $[28,29]$. Lin et al. also suggested to use the openEHR approach for modeling CDEs [30]. Though this approach provides a comprehensive structure with two-level modeling, several limitations when implementing openEHRs have been identified in various studies such as immaturity of archetype modification operations, insufficient support for hierarchical archetypes due to their granularity [31, 32], and the cost burden of development and adoption due to the complexity of defining openEHRs. Therefore, instead of utilizing external data models, we propose improving and extending the existing composite relationship by specifying two subtypes of aCDE, three subtypes of cCDEs, and four constraints to take advantage of utilizing CDEs and related technologies.

The newly released version of HL7 FHIR provides the ElementDefinition type, which is the core of the FHIR metadata layer and is closely (conceptually) aligned to ISO/IEC 11179. It has the result of mapping to the other standards as well to help implementers and clinical researchers understand the content and use it correctly. However, they found that the principles from both standards were totally different. FHIR does not differentiate the difference between a CDE and a CDE value and the FHIR specification is heavily type dependent. For instance, HL7 FHIR provides the pair of Questionnaire and QuestionnaireResponse resources and a pair of Appointment and AppointmentResponse resources at the same time. Also, the FHIR specification includes constraints and other concerns that are outside the scope of ISO/IEC 11179. Thus, the HL7 admitted that there still was a shortage of connection between HL7 FHIR and ISO/IEC 11179. It is said that the FHIR Infrastructure work group is considering rolling the DataElement resource into the StructureDefinition resource. If this is done, DataElement resource will be treated as a type of logical model (whether there will be a distinct 'type' for it is unclear) [33].

Since the FHIR specification includes concepts for the group and constraints, they were matched with our proposed concepts of composite and the part of constraints (ordered, operated). However, some of the semantic types and constraints that we have proposed are not provided by FHIR. We detailed whether our proposed semantic types and constraints were covered by FHIR. Since the FHIR Questionnaire is the only resource, which is related to clinical forms or documents, we distinguished from the other FHIR resources (Table 7).

\section{Overcoming the challenges of understanding semantic relationships of form-IEVeL data}

This paper has presented an in-depth evaluation of the ISO/IEC 11179 MDR standard based CDE semantic interrelationships in the context of formalizing clinical document structures. For converting form-level data into DE-level data, two cCDEs (repeated and dictionary cCDEs) and their related constraints were developed, which provide the following benefits:

\section{1) Repeated cCDEs support clinical data} management in a tabular format in a clinical document. Since multiple value sets are supported to be represented in a unified tabular format, a repeated $\mathrm{CCDE}$ is useful for managing sequential data entry in a tabular format and for analyzing how the values change over time. A repeated $\mathrm{CCDE}$ enables standard MDR-based CDE-level descriptions and evaluations of clinical data entry in a tabular format.

2) Dictionary cCDEs enable biomedical knowledge to be brought from a dictionary database via a variable aCDE. Data items referencing a certain standard terminology appear frequently on clinical forms. A dictionary cCDE can help to include rich semantics from externally managed biomedical terminologies and/or dictionaries, with rich attributes being applied for input data validation.

3) Four different types of constraints enable rich evaluations of input values. A prefix notation with functional logic programming can be applied for evaluating user-defined constraints in order to ensure contextual correctness and interrelationships among data items on clinical document.

\section{Advantages of using CDEs and CDE relationships for building clinical documents}

The data element is the atomic unit of data and is associated with a data element concept (DEC, an abstract unit of knowledge for representing semantics) and a value domain (representation of data including the data type and permissible values) according to the ISO/IEC 11179 MDR standard. The DEC is the combination of an object class (a set of entities) and a property (a peculiarity common to all member of an object class). As these two components of DEC are matched to the standard medical terminologies, it 
Table 7 Comparison of our proposed semantic types and constraints with the FHIR Questionnaire resource and the other FHIR resources

\begin{tabular}{|c|c|c|c|}
\hline \multicolumn{2}{|c|}{ CDE Semantic Type } & \multirow{2}{*}{$\begin{array}{l}\text { FHIR Questionnaire } \\
\text { No, it does not support the entry of multiple types of } \\
\text { data. }\end{array}$} & \multirow{2}{*}{$\begin{array}{l}\text { FHIR other resources } \\
\text { Not applicable, there is no restriction on the datatype as it is } \\
\text { represented JSON, XML. }\end{array}$} \\
\hline $\mathrm{aCDE}$ & Hybrid & & \\
\hline & Variable & Yes, it is supported by "coding". & Yes, it is supported by "coding". \\
\hline \multirow[t]{3}{*}{ CCDE } & General & $\begin{array}{l}\text { Yes, it is supported because the FHIR is following a } \\
\text { structured model. }\end{array}$ & $\begin{array}{l}\text { Yes, it is supported because the FHIR is following a structured } \\
\text { model. }\end{array}$ \\
\hline & Repeated & Yes, it is supported by "repeats". & $\begin{array}{l}\text { Yes, it is supported because the FHIR is } \\
\text { allowing repeated representation of the group of items. }\end{array}$ \\
\hline & Dictionary & $\begin{array}{l}\text { Not applicable, it does not support any value related } \\
\text { rule. }\end{array}$ & Not applicable, it does not support any value related rule. \\
\hline \multirow[t]{4}{*}{ Constraint } & Operated & Allowing only logical operations. & $\begin{array}{l}\text { Only resources that have the "operator" are supported (e.g., } \\
\text { Observation Resouce). }\end{array}$ \\
\hline & Required & Yes, it is supported by "required". & Yes, it is supported by "required". \\
\hline & Dependent & $\begin{array}{l}\text { Not applicable, it does not support any value related } \\
\text { rule. }\end{array}$ & Not applicable, it does not support any value related rule \\
\hline & Ordered & Although not explicit, it is included in the structure. & $\begin{array}{l}\text { Only resources that have "sequences" are supported (e.g., Claim } \\
\text { Resouce) }\end{array}$ \\
\hline
\end{tabular}

strengthens the semantic part. It is an advantage to use CDE. Our proposed new semantic types and constraints comply with this part in the ISO/IEC 11179 standard.

As verified in the evaluation part of this study, building clinical documents with CDEs can provide three major advantages. First, it prevents the generation of redundant data by facilitating predefined and registered CDEs to the MDR. Second, it ensures semantic data integrity since an MDR-based CDE has comprehensive and standardized metadata attributes for data description and the proposed $\mathrm{CCDE}$ provides a means to encode rich constraints for inter-CDE relationships. The health data of a patient that are fragmented, dispersed, and duplicated in a variety of clinical documents across different medical centers should be integrated, and mapping data items to CDEs facilitates data integration and semantic interoperability across different clinical documents. Third, clinical data exchange and sharing can be greatly facilitated by this approach.

\section{Limitation and future work}

The real-life clinical documents provide reasonable examples of reality, but particular instances of reality do not necessarily always provide good representative examples. For instance, we found that the quality of data in the clinical documents is dependent on whether the clinicians who wrote these documents were well trained in terminology representation to be inclusive in writing correctly and sufficiently valid clinical documents. If the document provides poor examples, then the outcome of the evaluation will also be poor. It is not only the problem of clinical documents but also it can be applied to when a clinical researcher creates data in the FHIR model or a physician inputs clinical data in an EHR system. Thus, we should measure the DQ, which is one of the aspects of the interoperability that reveals the process of standardizing EHRs to ensure the selected clinical documents are a good representation of the evaluation.

We also found that one essential issue was whether our proposed semantic types and constraints ensure semantical consistency with the use of standard biomedical terminologies. For the instance of data transfer and the purpose of interoperability, it is important to examine how well our proposed semantic types and constraints correspond to the standard biomedical terminologies and how we can address the issue of terminology variations. Although the DEC part of the ISO/IEC 11179 is matched to the standard medical terminologies, when multiple standard biomedical vocabularies are used in the complicated CDEs the above issue can occur. A similar issue can occur when we utilize the dictionary cCDE, since it includes a biomedical vocabulary. For instance, the dictionary cCDE can consider different 'versions' of a particular laboratory test with different time stamps, which could end with a differing variance of normal ranges. In other words, even if we reference the same standard vocabulary for the dictionary cCDE, the result could be different. We will measure another DQ for semantical consistency from the two issues mentioned above as a future work.

To measure DQ, we will consider the 5 different dimensions of DQ such as completeness, correctness, concordance, plausibility, and currency. The strategies used to assess the dimensions of DQ fell into seven DQ methods such as gold standard, data element agreement, element presence, data source agreement, distribution comparison, and validity check as a future work [34]. 


\section{Conclusion}

The sharing and understanding of data from multiple different domains can be facilitated by standardization. An MDR-based CDE is considered a type of standardized data with specified concept and value domains. However, ISO/IEC 11179 MDR-based CDEs do not provide the ability to describe constraints on a CDE or relationships among different CDEs, instead merely focusing on single independent CDEs, which makes it difficult to either correctly compose or interpret CDEs on clinical documents. We developed MDR-based extended semantic types and constraints, and it can facilitate comprehensive representation of clinical documents with rich semantics and improved semantic interoperability.

\section{Supplementary information}

Supplementary information accompanies this paper at https://doi.org/10. 1186/s12911-020-01168-0.

Additional file 1: Supplementary Table S1. List of NINDS CRFs with stroke CDEs related CRFs $(n=35)$, general CDEs related CRFs $(n=15)$, and common CRFs $(n=7)$. Supplementary Table S2. List of DialysisNet related Forms. Supplementary Table S3. List of general, dictionary, and repeated CCDEs with the numbers of operated, required, dependent, and ordered constraints extracted from five clinical documents used at five teaching hospitals in Korea. Supplementary Table S4. Distribution of aCDEs and CCDEs extracted from five clinical forms used at five teaching hospitals in Korea. Supplementary Table S5. List of 327 aCDEs comprising 20 cCDEs. The order of the CCDEs is identical to that in Supplementary Table S1. Supplementary Table S6 Distribution of aCDEs and cCDEs extracted from 14 FHIR resources of FHIR bulk sample data. List of unique 75 aCDEs comprising by 28 cCDEs from 238 aCDEs. The absence of repeated CCDE for some FHIR resources means that the configuration of aCDEs has been changed for each data.

Supplementary Table S7. List of 75 aCDEs comprising by 28 cCDEs. The order of the CCDEs is identical to that in Supplementary Table S4. Supplementary Table $\mathbf{S 8}$ List of categorized MIMIC-III database in which matched by aCDE, cCDE and constraints. Six tables are related hybrid and variable aCDE (23\%), four tables are related dictionary CCDE (15\%), and all tables are related to required constraints. Supplementary Table S9 List the detail elements of MIMIC-III database, which were matched to our proposed semantic types and constraints. Hybrid aCDEs in four tables, variable aCDE in four tables, operated constraint in two tables.

\section{Abbreviations \\ aCDE: Atomic CDE; BMI : Body mass index; CCDE: Composite CDE; CDE: Common data element; CDISC: Clinical data interchange standards consortium; CRF: Case report form; DDE: Derived data element; DE: Data element; DQ: Data Quality; EHR: Electronic health record; FHIR: Fast Healthcare Interoperability Resources; JSON : Javascript object notation; MDR: Metadata registry; MIMIC-III: Medical Information Mart for Intensive Care; NINDS: National institute of neurological disorders and stroke; ODM: Operational data model}

\section{Acknowledgements}

Authors thank the five institutions to generously provided their clinical document for common data element evaluations. Authors also thank the public opening of NINDS CDE Project and DialysisNet and Avatar Beans CDE Project as well as FHIR bulk sample data and the MIMIC-III demo dataset.

\section{Authors' contributions}

HHK and JHK designed the study and wrote the manuscript. YRP contributed to provide source data. YRP and SL performed manual curation and evaluation of CDEs and new CDE types. JHK supervised the project. All authors participated in the discussion of the study results and commented on the manuscript at all stages of manuscript preparation and revisions. The author(s) read and approved the final manuscript.

\section{Funding}

This work was supported by a grant of the Korean Health Technology R\&D Project, Ministry of Health and Welfare, Republic of Korea (HI18C2386).

\section{Availability of data and materials}

Not Applicable.

\section{Ethics approval and consent to participate}

Not Applicable. To give you more description, we have not used any of patients' data. The data described in the Methods section are metadata, which is data about data including data 'specifications' and 'definitions.' We have had no chance of using patients' private and/or personal information at all in writing the manuscript.

Consent for publication

Not Applicable.

\section{Competing interests}

None of the authors has conflicts of interest with other persons or organizations that could inappropriately influence their work.

\section{Author details}

${ }^{1}$ Seoul National University Biomedical Informatics (SNUBI), Division of Biomedical Informatics, Seoul National University College of Medicine, Seoul 03080, Republic of Korea. ${ }^{2}$ Department of Biomedical Systems Informatics, Yonsei University College of Medicine, Seoul 03722, Republic of Korea. ${ }^{3}$ Department of Biomedical Informatics, College of Medicine, Konyang University, Daejeon 35365, Republic of Korea.

Received: 12 June 2019 Accepted: 25 June 2020

Published online: 03 July 2020

\section{References}

1. Richesson RL, Krischer J. Data standards in clinical research: gaps, overlaps, challenges and future directions. J Am Med Inform Assoc. 2007. https://doi. org/10.1197/jamia.M2470.

2. Ferranti JM, Musser RC, Kawamoto K, Hammond WE. The clinical document architecture and the continuity of care record: a critical analysis. J Am Med Inform Assoc. 2006. https://doi.org/10.1197/jamia.M1963.

3. Mohanty SK, Mistry AT, Amin W, et al. The development and deployment of common data elements for tissue banks for translational research in cancer-an emerging standard based approach for the mesothelioma virtual tissue Bank. BMC Cancer. 2008. https://doi.org/10.1186/1471-2407-8-91.

4. Groft SC, Rubinstein YR. New and evolving rare diseases research programs at the National Institutes of Health. Public Health Genomics. 2013. https:// doi.org/10.1159/000355929.

5. NIH Common Data Element (CDE) Repository Website. https://www.nIm.nih. gov/cde/. Accessed 20 Mar 2020.

6. DE and CDE definition in NIM. Website. https://www.nlm.nih.gov/cde/ glossary.html\#cdedefinition. Accessed 12 Mar 2020.

7. Saver IL, Warach S, Janis S, et al. Standardizing the structure of stroke clinical and epidemiologic research data: the National Institute of Neurological Disorders and Stroke (NINDS) stroke common data element (CDE) project. Stroke. 2012. https://doi.org/10.1161/STROKEAHA.111.634352.

8. Newton KM, Peissig PL, Kho AN, Bielinski SJ, Berg RL, Choudhary V, Basford M, Chute CG, Kullo IJ, Li R, Pacheco JA, Rasmussen LV, Spangler L, Denny JC. Validation of electronic medical record-based phenotyping algorithms: results and lessons learned from the eMERGE network. J Am Med Inform Assoc. 2013. https://doi.org/10.1136/amiajnl-2012-000896.

9. Nahm M, Walden A, McCourt B, et al. Standardising clinical data elements. Int J Funct Inform Personal Med. 2010. https://doi.org/10.1504/IJFIPM.2010 040213

10. Park YR, Yoon YJ, Kim HH, Kim JH. Establishing semantic interoperability of biomedical metadata registries using extended semantic relationships. Stud Health Technol Inform. 2013;192:618-21.

11. Nadkarni PM, Brandt CA. The common data elements for cancer research: remarks on functions and structure. Methods Inf Med. 2006;45:594-601. 
12. Richesson RL, Nadkarni P. Data standards for clinical research data collection forms: current status and challenges. J Am Med Inform Assoc. 2011. https:// doi.org/10.1136/amiajnl-2011-000107.

13. ISO/IEC 11179. International Standard, International Electrotechnical Commission, Information technology - Metadata registries (MDR) — Part 3: Registry metamodel and basic attributes. https://webstore.iec.ch/preview/ info_isoiec11179-3\%7Bed3.0\%7Den.pdf, Publication date April 10, 2006.

14. NCI caDSR Wiki, CDE Curation Tool User Guide- Creating Derived Data Element. Website. https://wiki.nci.nih.gov/display/caDSR/10+-+Creating+ Derived+Data+Elements/. Accessed 20 Mar 2020.

15. Data type in Wikipedia. Website. https://en.wikipedia.org/wiki/Data_type/. Accessed 12 Mar 2020

16. NINDS Common Data Elements Website. https://commondataelements. ninds.nih.gov/. Accessed 12 Mar 2020.

17. Ku HS, Kim S, Kim H, Kim JH. DialysisNet: application for integrating and management data sources of hemodialysis information by continuity of care record. Healthc Inform Res. 2014. https://doi.org/10.4258/hir.2014.20.2. 145

18. Park $Y R, K i m ~ H, A n E Y$, et al. Establishing semantic interoperability in the course of clinical document exchange using international standard for metadata registry. J Korean Med Assoc. 2012. https://doi.org/10.5124/jkma. 2012.55.8.729.

19. Kim JH. Health avatar: an informatics platform for personal and private big data. Healthc Inform Res. 2014. https://doi.org/10.4258/hir.2014.20.1.1.

20. Braunstein ML. Healthcare in the age of interoperability: the promise of fast healthcare interoperability resources. IEEE Pulse. 2018. https://doi.org/10. 1109/MPUL.2018.2869317.

21. Braunstein ML. Health Care in the age of interoperability part 6: the future of FHIR. IEEE Pulse. 2019. https://doi.org/10.1109/MPULS.2019.2922575.

22. FHIR Bulk downloader sample app. Website. https://bulk-data.smarthealthit. org/sample-app/index.html. Accessed Mar. 20, 2020.

23. HL7 FHIR version 4.0 Resource List. Website. https://www.hl7.org/fhir/ resourcelist.html. Accessed Mar. 20, 2020,

24. Johnson A, Pollard T, Mark R. MIMIC-III Clinical Database Demo (version 1.4). PhysioNet. 2019; https://doi.org/10.13026/C2HM2O

25. MIMIC-III Critical Care Database. Website. https://mimic.physionet.org/about/ mimic/. Accessed Mar. 20, 2020

26. NINDS Common Data Elements. Website. https://www commondataelements.ninds.nih.gov/Doc/Stroke/F1168_Laboratory_Tests_ Permissible_Values_for_Stroke.xlsx. Accessed Mar. 20, 2020.

27. Wikipedia. Website. https://en.wikipedia.org/wiki/Polish_notation. Accessed Mar. 20, 2020

28. Ngouongo SM, Löbe M, Stausberg J. The ISO/IEC 11179 norm for metadata registries: does it cover healthcare standards in empirical research? J Biomed Inform. 2013. https://doi.org/10.1016/j.jbi.2012.11.008.

29. Iberson-Hurst D. The CDISC operational data model: ready to roll? Appl Clin Trials. 2004;13:48-53.

30. Lin CH, Fann YC, Liou DM. An exploratory study using an openEHR 2-level modeling approach to represent common data elements. J Am Med Inform Assoc. 2016. https://doi.org/10.1093/jamia/ocv137.

31. Garde S, Hovenga E, Buck J, Knaup P. Expressing clinical data sets with openEHR archetypes: a solid basis for ubiquitous computing. Int I Med Inform. 2007. https://doi.org/10.1016/j.jimedinf.2007.02.004.

32. Späth MB, Grimson J. Applying the archetype approach to the database of a biobank information management system. Int J Med Inform. 2011. https:// doi.org/10.1016/j.jimedinf.2010.11.002.

33. HL7 DataElement resource. Website. https://hl7.org/fhir/STU3/dataelement html. Accessed Mar. 20, 2020.

34. Weiskopf NG. Weng C. Methods and dimensions of electronic health record data quality assessment: enabling reuse for clinical research. J Am Med Inform Assoc. 2013. https://doi.org/10.1136/amiajnl-2011-000681.

\section{Publisher's Note}

Springer Nature remains neutral with regard to jurisdictional claims in published maps and institutional affiliations.

Ready to submit your research? Choose BMC and benefit from:

- fast, convenient online submission

- thorough peer review by experienced researchers in your field

- rapid publication on acceptance

- support for research data, including large and complex data types

- gold Open Access which fosters wider collaboration and increased citations

- maximum visibility for your research: over $100 \mathrm{M}$ website views per year

At $\mathrm{BMC}$, research is always in progress.

Learn more biomedcentral.com/submissions 